\title{
Performance Analysis of Selective Decode-and-Forward Multinode Incremental Relaying with Maximal Ratio Combining
}

\author{
Amir Hadjtaieb ${ }^{1}$, Ali Chelli ${ }^{1}$, Mohamed-Slim Alouini ${ }^{1}$, and Hatem Boujemaa ${ }^{2}$ \\ ${ }^{1}$ King Abdullah University of Science and Technology (KAUST), Thuwal, Makkah Province, Saudi Arabia. \\ ${ }^{2}$ Higher School of Communications of Tunis, University of Carthage, Tunisia. \\ Emails: \{amir.hadjtaieb, ali.chelli, slim.alouini\}@kaust.edu.sa, boujemaa.hatem@supcom.rnu.tn
}

\begin{abstract}
In this paper, we propose an incremental multinode relaying protocol with arbitrary $N$-relay nodes that allows an efficient use of the channel spectrum. The destination combines the received signals from the source and the relays using maximal ratio Combining (MRC). The transmission ends successfully once the accumulated signal-to-noise ratio (SNR) exceeds a predefined threshold. The number of relays participating in the transmission is adapted to the channel conditions based on the feedback from the destination. The use of incremental relaying allows obtaining a higher spectral efficiency. Moreover, the symbol error probability (SEP) performance is enhanced by using MRC at the relays. The use of MRC at the relays implies that each relay overhears the signals from the source and all previous relays and combines them using MRC. The proposed protocol differs from most of existing relaying protocol by the fact that it combines both incremental relaying and MRC at the relays for a multinode topology. Our analyses for a decode-and-forward mode show that: (i) compared to existing multinode relaying schemes, the proposed scheme can essentially achieve the same SEP performance but with less average number of time slots, (ii) compared to schemes without MRC at the relays, the proposed scheme can approximately achieve a $3 \mathrm{~dB}$ gain.
\end{abstract}

\section{INTRODUCTION}

Cooperative diversity is a promising technique that allows to mitigate fading effects and to provide high data rate services in wireless communication by the use of relays [1]-[4].

In the presence of multiple relays, several relaying schemes have been utilized in the literature. In [5], the authors studied the performance of multihop transmission, where each relay forwards the received signal from the previous relay to the next one. In [6] and [7], an aggregation of a single-hop with $N$ two-hop transmissions is used. This strategy can achieve a full diversity order. However, this scheme requires the use of $N+1$ orthogonal channels which results in a low spectral efficiency.

To achieve a higher spectral efficiency, incremental relaying can be exploited [1], [8], [9]. In such strategy, the number of relays contributing to the transmission is increased sequentially. In each step, the destination tests if the accumulated SNR from the source and a subset of the relays is sufficiently high. If the test is positive, a feedback from the destination indicates the success of the transmission, and the remaining relays keep idle. It has to be mentioned that the number of time slots used in incremental relaying is adapted to the channel conditions which increases the spectral efficiency of this scheme. Our work differs from [8] and [9] in two major aspects. Firstly, we consider in our study a decode-andforward (DF) mode as opposed to the amplify-and-forward (AF) mode investigated in [8] and [9]. It is well known that the performance analyses of $\mathrm{AF}$ schemes are substantially different from that of DF schemes. Secondly, in [8] and [9] the relays that should transmit in the subsequent time slots remain inactive while other relays are transmitting. In our scheme, these relays overhear the transmission of all previous relays and combine all the replicas that they overheard over several time slots using maximal ratio combining (MRC). By using MRC at the relays, the probability of successful decoding at the relays increases and the bit error probability (BEP) at the destination decreases.

Sadek et al. proposed in [10] a selective multinode relaying (SMR) scheme where MRC at the relays is utilized. In this relaying strategy, no incremental relaying is used which results in a loss in the spectral efficiency. In this paper, we combine both incremental relaying at the destination with MRC at the relays to improve both the spectral efficiency and the BEP performance. The proposed protocol is called selective multinode incremental relaying (SMIR). The selective relaying rule impose that: "The relay forward the message only if the total received SNR at the relay exceeds a predefined threshold. Otherwise, it remains idle". Analytical expressions for the symbol error probability (SEP) are provided for an arbitrary $N$-node network employing $\mathrm{M}$-ary phase shift keying (MPSK) modulation. We show that the use of MRC at the relays allows improving the SEP performance by approximately $3 \mathrm{~dB}$. Furthermore, we provide exact expressions for the average number of time slots $\overline{N_{p}}$ required to send a data burst.

The remainder of this paper is organized as follows. Section II introduces the system model under investigation. Sections III provides exact expressions of the SEP for the proposed scheme over Rayleigh fading channels. In Section IV, the average number of time slots $\overline{N_{p}}$ required to send a data burst is evaluated. Finally, Section V offers some concluding remarks. 


\section{System Model ANd Protocol Description}

We consider an arbitrary $N$-relay wireless network. The wireless link between any two nodes is modeled as a slow and frequency-flat fading channel with additive white Gaussian noise (AWGN). The cooperation strategy we are considering employs a selective DF protocol and the MRC technique at the relay nodes. Moreover, we assume that the relay can judge whether the message is correctly decoded or not. Consequently, it forwards the message if it is correctly decoded; otherwise, it remains idle. This assumption is shown in [10] to be very close to the practical scenario of comparing the received SNR to a given threshold.

The cooperation protocol has a maximal number of $(N+1)$ phases. It has to be noted that the number of phases used to transmit a message varies depending on the channel conditions. The transmission terminates if the total received SNR at destination is sufficiently high $(\mathrm{SNR}>\xi)$ for successful decoding. For notation convenience, we begin with phase 0 . The protocol is depicted in Fig. 1.

(a)

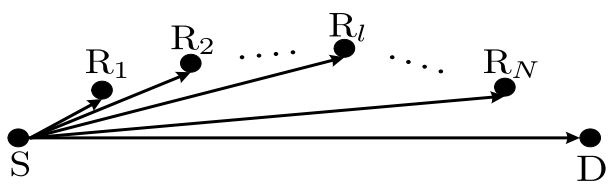

(b)

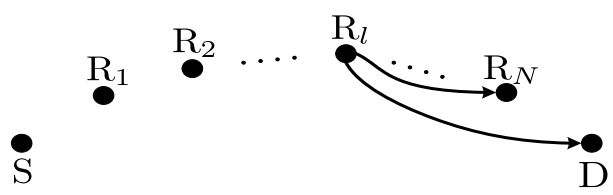

Fig. 1. Illustration of the protocol:(a) phase 0 and (b) phase $l$.

- Phase 0: the source broadcasts the signal as shown in Fig. 1.a. If the source-destination SNR is sufficiently high $(\mathrm{SNR}>\xi)$, a positive feedback message indicates the success of direct transmission and the source sends the next packet. Otherwise, a negative feedback implies that another replica of the signal should be sent in the next phase.

- Phase $l, 1 \leq l \leq(N-1)$ : the $l$ th relay combines the received signals from the source and the previous $(l-1)$ relays using MRC. If relay $l$ decodes correctly the message, it transmits the data packet to the destination and the following relays with power $P_{l}$ as shown in Fig. 1.b. The destination coherently combines all the received signals using MRC. If the SNR is sufficiently high, a feedback indicates the success of transmission after $l$ steps. Otherwise, a feedback requests another replica of the signal and we move to phase $l+1$.

- Phase $N$ : the $N$ th relay sends the message to the destination if it decoded the received message correctly. The destination performs the decoding based on all received replicas.

It has to be noted that our work differs from [9] and [8] by using a selective DF protocol instead of an AF scheme. In addition, in our scheme, we use MRC at the relays which allows improving the BEP performance at the destination.

The received signal at the destination from the source, $y_{S, D}$, and the $i$ th relay, $y_{S, R_{i}}$, can be written as

$$
\begin{aligned}
y_{S, D} & =\sqrt{P_{0}} h_{S, D} x+n_{S, D} \\
y_{S, R_{i}} & =\sqrt{P_{0}} h_{S, R_{i}} x+n_{S, R_{i}}, \quad 1 \leq i \leq N,
\end{aligned}
$$

where $P_{0}$ is the power transmitted at the source, $x$ is the transmitted symbol with unit power, $h_{S, D} \sim \mathrm{CN}\left(0, \sigma_{S, D}^{2}\right)$ and $h_{S, R_{i}}$ $\sim \mathrm{CN}\left(0, \sigma_{S, R_{i}}^{2}\right)$ are the channel fading coefficients between the source and the destination, and $i$ th relay, respectively. The term $\mathrm{CN}\left(\alpha, \sigma^{2}\right)$ denotes a circularly symmetric complex Gaussian random variables with mean $\alpha$ and variance $\sigma^{2}$. The terms $n_{S, D}$ and $n_{S, R_{i}}$ denote the AWGN. The received signal at the relay can be expressed as

$$
y_{R_{l}}=\sqrt{P_{0}} h_{S, R_{l}}^{*} y_{S, R_{l}}+\sum_{i=1}^{l-1} \sqrt{\hat{P}_{i}} h_{R_{i}, R_{l}}^{*} y_{R_{i}, R_{l}},
$$

where $h_{R_{i}, R_{l}} \sim C N\left(0, \sigma_{R_{i}, R_{l}}^{2}\right)$ is the channel fading coefficient between the $i$ th and the lth relays. In (2), $y_{R_{i}, R_{l}}$ denotes the signal received at the $l$ th relay from the $i$ th relay, and can be modeled as

$$
y_{R_{i}, R_{l}}=\sqrt{\hat{P}_{i}} h_{R_{i}, R_{l}} x+n_{R_{i}, R_{l}},
$$

where $\hat{P}_{i}$ is the power transmitted at relay $i$, and $\hat{P}_{i}=P_{i}$ if relay $i$ correctly decodes the transmitted message; otherwise, $\hat{P}_{i}=0$. The $l$ th relay uses $y_{R_{l}}$ in (2) as the detection statistics. Finally, the received signal at the destination can be modeled as

$$
y_{D}=\sqrt{P_{0}} h_{S, D}^{*} y_{S, D}+\sum_{i=1}^{l} \cdot \sqrt{\hat{P}_{i}} h_{R_{i}, D}^{*} y_{R_{i}, D} .
$$

In all the cooperation scenarios considered, the total transmitted power is fixed as $P_{0}+\sum_{i=1}^{N} P_{i}=P$.

\section{ERror PERformance ANAlysis}

\section{A. General SEP Expression}

In this section, we present an SEP performance analysis of the proposed SMIR scheme for system utilizing MPSK modulation. In a given phase $l \geq 1$, the network can take $2^{l}$ states. Each relay can be in one of two states: 1 if it decodes correctly or 0 if not. Hence, we can define a $1 \times l$ vector $S_{l}$ to represent the state of the $l$ first relays. The $k$ th element of the vector $S_{l}$ equals to 1 if the $k$ th relay decodes correctly and 0 if not. We define also $B_{x, n}$ a $1 \times n$ binary representation of a decimal number $x$, with $B_{x, n}[1]$ the most significant bit. Note that $x$ can take values from 0 to $2^{l}-1$. So, $S_{l}=B_{x, l}$ implies that, in the phase $l$, the $k$ th relay is in state $S_{l}[\mathrm{k}]=B_{x, l}[\mathrm{k}]$.

Conditioning on the phase and the network state, the probability of error at the destination given the channel state information (CSI) can be expressed using Bayes rule as follows

$$
\begin{aligned}
& P_{e \mid C S I}=\operatorname{Pr}(e \mid \text { phase }=0) \operatorname{Pr}(\text { phase }=0) \\
& +\sum_{l=1}^{N} \sum_{i=0}^{2^{l}-1} \operatorname{Pr}\left(e \mid S_{l}=B_{i, l}, \text { phase }=l\right) \\
& \times \operatorname{Pr}\left(S_{l}=B_{i, l} \mid \text { phase }=l\right) \operatorname{Pr}(\text { phase }=l),
\end{aligned}
$$


where $e$ denotes the event decoding error at the destination. In the second term of (5), the first summation is over the $N$ phases whereas the second summation is over all possible states of the the first $l$ relays at phase $l$.

For a given phase $l$ and a given network state $S_{l}=B_{i, l}$, the conditional SEP at the destination can be expressed as

$$
\operatorname{Pr}\left(e \mid S_{l}=B_{i, l}, \text { phase }=l\right)=\Psi\left(\gamma_{l}\right),
$$

where $\gamma_{l}$ is the total received SNR at the destination which can be calculated as follows

$$
\gamma_{l}=\frac{P_{0}\left|h_{S, D}\right|^{2}+\sum_{j=1}^{l} P_{j} B_{i, l}[j]\left|h_{R_{j}, D}\right|^{2}}{N_{0}} .
$$

It is obvious that this expression is different from the instantaneous SNR of AF strategy defined in [11, Eq.(4)].

If an MPSK modulation is used, the $\operatorname{SEP} \psi_{P S K}(\gamma)$ can be expressed as [12]

$$
\Psi_{P S K}(\gamma) \triangleq \frac{1}{\pi} \int_{0}^{(M-1) \pi / M} \exp \left(-\frac{b_{P S K} \gamma}{\sin ^{2}(\theta)}\right) d \theta,
$$

where $b_{P S K}=\sin ^{2}(\pi / M)$.

Now, let us compute the second term of the double summation in (5). The state of each relay depends on its SNR which depends on the number of relays that decode correctly. Thus, the state of each relay depends on the state of the previous relays. Conditioning on the phase, the probability that the network is in a given state is expressed as follows

$$
\begin{aligned}
& \operatorname{Pr}\left(S_{l}=B_{i, l} \mid \text { phase }=l\right)=\operatorname{Pr}\left(S_{l}[1]=B_{i, l}[1]\right) \times \\
& \operatorname{Pr}\left(S_{l}[2]=B_{i, l}[2] \mid S_{l}[1]=B_{i, l}[1]\right) \times \ldots \times \\
& \operatorname{Pr}\left(S_{l}[l]=B_{i, l}[l] \mid S_{l}[l-1]=B_{i, l}[l-1], \ldots, S_{l}[1]=B_{i, l}[1]\right) .
\end{aligned}
$$

The conditional probability that the $k$ th relay is in state $B_{i, l}[\mathrm{k}]$ given the state of the previous relays can be expressed as follows

$$
\begin{aligned}
P_{k, i}^{l} & \triangleq \operatorname{Pr}\left(S_{l}[k]=B_{i, l}[k] \mid S_{l}[k-1]=B_{i, l}[k-1],\right. \\
& \left.\ldots, S_{l}[1]=B_{i, l}[1]\right) \\
& = \begin{cases}\Psi\left(\gamma_{R_{k}}\right), & \text { if } B_{i, l}[\mathrm{k}]=0 \\
1-\Psi\left(\gamma_{R_{k}}\right), & \text { if } B_{i, l}[\mathrm{k}]=1,\end{cases}
\end{aligned}
$$

where $\gamma_{R_{k}}$ is the total received SNR at the $k$ th relay computed as follows

$$
\gamma_{R_{k}}=\frac{P_{0}\left|h_{S, R_{k}}\right|^{2}+\sum_{j=1}^{k-1} P_{j} B_{i, l}[j]\left|h_{R_{j}, R_{k}}\right|^{2}}{N_{0}} .
$$

The last part in the calculation is the probability that transmission terminates successfully at phase $l$ which is the third term in the double summation in (5). The probability of successful decoding at destination in phase $l$ can be expressed as follows

$$
\begin{aligned}
& \operatorname{Pr}(\text { phase }=l) \\
& =\left\{\begin{array}{c}
\operatorname{Pr}\left(\gamma_{S D}>\xi\right)=1-\mathrm{F}_{\gamma_{S D}}(\xi), \\
\text { if phase }=0 \\
\operatorname{Pr}\left(\gamma_{l}>\xi, \gamma_{l-1}<\xi\right)=\mathrm{F}_{\gamma_{l-1}}(\xi)-\mathrm{F}_{\gamma_{l}}(\xi), \\
\text { if phase }=1, \ldots,(N-1) \\
\operatorname{Pr}\left(\gamma_{N-1}<\xi\right)=\mathrm{F}_{\gamma_{N-1}}(\xi), \\
\text { if phase }=N .
\end{array}\right.
\end{aligned}
$$

where $\mathrm{F}_{X}$ is the $\mathrm{CDF}$ of the random variable $\mathrm{X}$.

We can pass now to compute the average SEP, we need to average the conditional SEP in (5) over all channel realizations. Using (6), (9), (10) and (12), the average SEP at destination can be expressed as follows

$$
\begin{aligned}
& P_{S E P}=E_{C S I}\left\{P_{e \mid C S I}\right\}=\operatorname{Pr}\left(\gamma_{S D}>\xi\right) E\left\{\Psi\left(\gamma_{S D}\right)\right\} \\
& +\sum_{l=1}^{N-1} \sum_{i=0}^{2^{l}-1} \operatorname{Pr}\left(\gamma_{l}>\xi, \gamma_{l-1}<\xi\right) \times E\left\{\Psi\left(\gamma_{l}\right)\right\} \prod_{k=1}^{l} E\left\{P_{k, i}^{l}\right\} \\
& +\sum_{i=0}^{2^{N}-1} \operatorname{Pr}\left(\gamma_{N-1}<\xi\right) E\left\{\Psi\left(\gamma_{N}\right)\right\} \prod_{k=1}^{N} E\left\{P_{k, i}^{N}\right\} .
\end{aligned}
$$

It has to be noted that we separated the quantities inside the expectation operator since they are function of independent random variables. This is due to the independence of the fading between any two nodes of the network. The terms $E\left\{\Psi\left(\gamma_{S D}\right)\right\}, E\left\{\Psi\left(\gamma_{l}\right)\right\}, E\left\{\Psi\left(\gamma_{N}\right)\right\}, E\left\{P_{k, i}^{l}\right\}$ can be written as

$$
\begin{gathered}
E\left\{\Psi\left(\gamma_{S D}\right)\right\}=\int_{0}^{\infty} \Psi(\gamma) f_{\gamma_{S D}}\left(\gamma \mid \gamma_{S D}>\xi\right) d \gamma \\
E\left\{\Psi\left(\gamma_{l}\right)\right\}=\int_{0}^{\infty} \Psi(\gamma) f_{\gamma_{l}}\left(\gamma \mid \gamma_{l-1}<\xi, \gamma_{l}>\xi\right) d \gamma \\
1 \leq l \leq N-1 \\
E\left\{\Psi\left(\gamma_{N}\right)\right\}=\int_{0}^{\infty} \Psi(\gamma) f_{\gamma_{N}}\left(\gamma \mid \gamma_{N-1}<\xi\right) d \gamma \\
E\left\{P_{k, i}^{l}\right\}=\int_{0}^{\infty} P_{k, i}^{l} f_{\gamma_{R_{k}}}(\gamma) d \gamma,
\end{gathered}
$$

where $f_{\gamma_{l}}(\gamma)(l=0, \ldots, N)$ is the probability density function (PDF) of the random variables $\gamma_{l}$. The term $f_{\gamma_{S D}}\left(\gamma \mid \gamma_{S D}>\xi\right)$ is the conditional PDF of $\gamma_{S D}$ given that $\gamma_{S D}$ is greater than the threshold $\xi$, while $f_{\gamma_{l}}\left(\gamma \mid \gamma_{l-1}<\xi\right)$ is the conditional PDF of $\gamma_{l}$ given that $\gamma_{l-1}$ is less than the threshold $\xi$. It has to be noted that the use of the moment generating function (MGF)-based approach [12] allows to simplify the double integral in (15), (16), (17), and (18) into a single integral if we replace $\Psi\left(\gamma_{l}\right)$ by its expression in (8).

\section{B. SEP Expression for Rayleigh Fading Channel}

The aim of this section is the evaluation of the SEP over Rayleigh fading channels. In this case, the SNR between each two nodes is an exponentially distributed random variable. So, $\gamma_{l}, 0 \leq l \leq N$ is the sum of independent and non-identically distributed (i.ni.d.) exponential random variables. 
Let $\mathcal{R}=\left\{R_{1}, \ldots, R_{p}, \ldots, R_{m}\right\}$ denote the set of relays that forwarded the signal to the destination at step $l$ and let $\lambda_{p}(p=$ $1, \ldots, m)$ stands for the SNR of the link between the relay $R_{p}$ and the destination. It follows that the equality $\lambda_{l}=\gamma_{S D}+$ $\sum_{k=1}^{l} B_{i, l}[k] \gamma_{R_{k} D}=\lambda_{0}+\sum_{p=1}^{m} \lambda_{p}$ holds, where $\lambda_{0}=\gamma_{S D}$. The PDF of $\gamma_{l}$ can be expressed as [13, Eq.(14.5-26)]

$$
f_{\gamma_{l}}(\gamma)=\sum_{j=0}^{m} \frac{C_{j}}{\bar{\lambda}_{j}} \exp \left(-\frac{\gamma}{\bar{\lambda}_{j}}\right)
$$

where $C_{j}=\prod_{k=0, k \neq j}^{m} \frac{\bar{\lambda}_{j}}{\lambda_{j}-\bar{\lambda}_{k}}$ and $\bar{\lambda}_{i}=E\left(\lambda_{i}\right)$.

For the case of a Rayleigh fading channel, using (12), we determine the exact expression of the probability of successful decoding at destination in phase $l \operatorname{Pr}($ phase $=l)$ as

$$
\begin{aligned}
& \operatorname{Pr}(\text { phase }=l) \\
& =\left\{\begin{array}{lr}
\exp \left(-\frac{\xi}{\bar{\gamma}_{S D}}\right) & \text { if phase }=0 \\
\sum_{j=0}^{m-1} \frac{\lambda_{m}}{\lambda_{j}} C_{j}\left(\exp \left(-\frac{\xi}{\lambda_{j}}\right)-\right. & \left.\exp \left(-\frac{\xi}{\lambda_{m}}\right)\right) \mathbf{1}_{\left(B_{i, l}[l]=1\right)}, \\
\sum_{j=0}^{m} C_{j}\left(1-\exp \left(-\frac{\xi}{\lambda_{j}}\right)\right), & \text { if phase }=1, \ldots,(N-1)
\end{array}\right.
\end{aligned}
$$

where $\mathbf{1}_{\left(B_{i, l}[l]=1\right)}$ equals to 1 when $\left(B_{i, l}[l]=1\right)$ and 0 otherwise.

Next, we compute the conditional PDFs $f_{\gamma_{S D}}\left(\gamma \mid \gamma_{S D}\right)$, $f_{\gamma_{l}}\left(\gamma \mid \gamma_{l-1}<\xi, \gamma_{l}>\xi\right)$ and $f_{\gamma_{N}}\left(\gamma \mid \gamma_{N-1}<\xi\right)$. These PDFs are required in (15), (16), and (17). Beginning with (15), the conditional PDF $f_{\gamma_{S D}}\left(\gamma \mid \gamma_{S D}>\xi\right)$ can be found as [14]

$$
f_{\gamma_{S D}}\left(\gamma \mid \gamma_{S D}>\xi\right)= \begin{cases}0, & \text { if } \gamma \leq \xi \\ \frac{\exp \left(\frac{\xi}{\bar{\gamma}_{S D}}\right)}{\bar{\gamma}_{S D}} \exp \left(\frac{-\gamma}{\bar{\gamma}_{S D}}\right), & \text { if } \gamma>\xi .\end{cases}
$$

In (16), the conditional PDF $f_{\gamma_{l}}\left(\gamma \mid \gamma_{l-1}<\xi, \gamma_{l}>\xi\right)(l=$ $1, \ldots, N-1)$ can be expressed as

$$
f_{\gamma_{l}}\left(\gamma \mid \gamma_{l-1}<\xi, \gamma_{l}>\xi\right)=\frac{f_{\gamma_{l}}\left(\gamma, \gamma_{l-1}<\xi, \gamma_{l}>\xi\right)}{P\left(\gamma_{l-1}<\xi, \gamma_{l}>\xi\right)} .
$$

The denominator of (22) is given by (20) and the numerator is given by the second case of (24).

In (17), the conditional PDF $f_{\gamma_{N}}\left(\gamma \mid \gamma_{N-1}<\xi\right)$ can be expressed as follows

$$
f_{\gamma_{N}}\left(\gamma \mid \gamma_{N-1}<\xi\right)=\frac{f_{\gamma_{N}}\left(\gamma, \gamma_{N-1}<\xi\right)}{P\left(\gamma_{N-1}<\xi\right)} .
$$

The denominator of (23) is given by (20) and the numerator can be obtained from (24) if we replace $l$ by $N$. The joint PDF $f_{\gamma_{l}}\left(\gamma, \gamma_{l-1}<\xi\right)$ reads as

$$
\begin{aligned}
& f_{\gamma_{l}}\left(\gamma, \gamma_{l-1}<\xi\right)= \\
& \begin{cases}f_{\gamma_{l}}(\gamma)=f_{\gamma_{l}}(\gamma)=\sum_{j=0}^{m} \frac{C_{j}}{\lambda_{j}} \exp \left(-\frac{\gamma}{\lambda_{j}}\right), & \text { if } \gamma \leq \xi \\
\sum_{j=0}^{m-1} \frac{C_{j}}{\lambda_{j}}\left(\exp \left(-\xi\left(\frac{1}{\lambda_{j}}-\frac{1}{\lambda_{m}}\right)\right)-1\right) \exp \left(-\frac{\gamma}{\lambda_{m}}\right) \mathbf{1}_{\left(B_{i, l}[l]=1\right)}, & \text { if } \gamma>\xi\end{cases}
\end{aligned}
$$

The proof of (24) is provided in the Appendix. Finally, (18) can be computed using the expression of $f_{\gamma_{R_{k}}}(\gamma)$ given by (19).

\section{Numerical Examples}

As a numerical example, Fig. 2 depicts the BEP versus SNR performance of the proposed SMIR scheme for different threshold values $\xi$. In this figure, we consider a BPSK modulation scheme and a number of relays equal to 3 . As shown

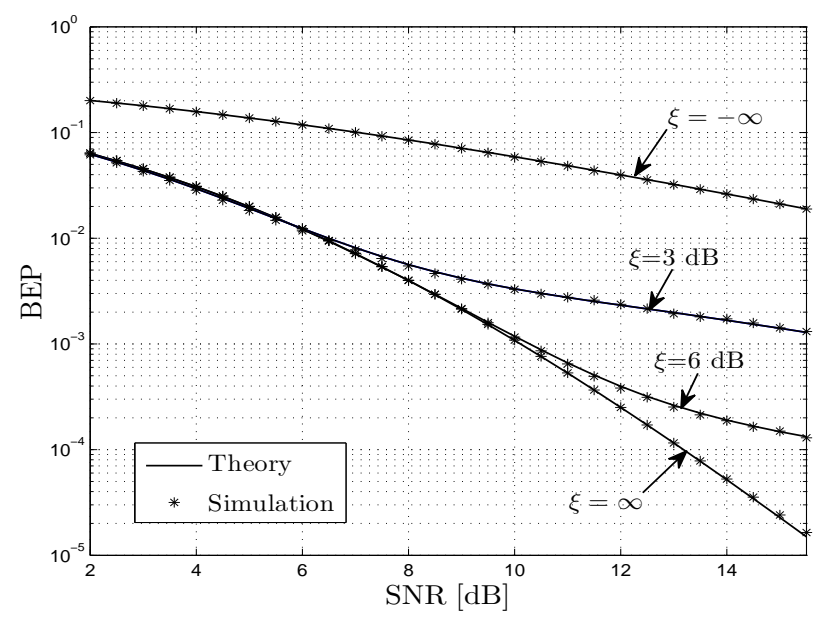

Fig. 2. BEP versus SNR of the proposed SMIR scheme for different threshold values $\xi$. The number of relays equals to 3 and the modulation scheme is BPSK.

in Fig. 2, the performance curves are upper-bounded by the BEP of the direct link $(\xi=-\infty)$ and lower-bounded by the BEP of the SMR scheme $(\xi=\infty)$ introduced by Sadek et $a l$. in [10]. If $\xi$ has a small value, we have a higher BEP. However in the same time, the channel resources (frequency and time) are saved. On the other hand, when $\xi$ has a large value, we have a better performance, but we use more channel resources. Therefore, there is a trade-off between performance and spectral efficiency. So, $\xi$ must be chosen after taking into account the type of application offered by the communication system as well as the quality of service required by the user.

Fig. 3 illustrates the BEP versus SNR performance of the proposed SMIR scheme for different number of relays and a fixed threshold $\xi=5 \mathrm{~dB}$ for a BPSK modulation scheme. This figure shows that given a fixed threshold, we have interest to increase the number of relays to get a more reliable communication. Thus, for a fixed threshold $\xi$, the greater network we have, the better performance we obtain.

To demonstrate the benefit of using MRC at relays, we plot the BEP versus SNR of SMIR scheme with and without MRC at the relays in Fig. 4. For this figure, the channel variance depends on the distance $d$ and propagation path loss $\alpha$ as follows $\sigma^{2} \propto d^{-\alpha}$ where $\alpha=3$. This figure shows that the gain that we achieve by using MRC at relays is approximately equal to $3 \mathrm{~dB}$ at a BEP of $10^{-2}$. This result confirms the benefit of using MRC at relays. Note that the BEP analysis of the SMIR scheme without MRC at the relays is a special case of the SMIR scheme with MRC. Due to space limitation, the analysis of the SMIR scheme without MRC at the relays is not been included in this paper. 


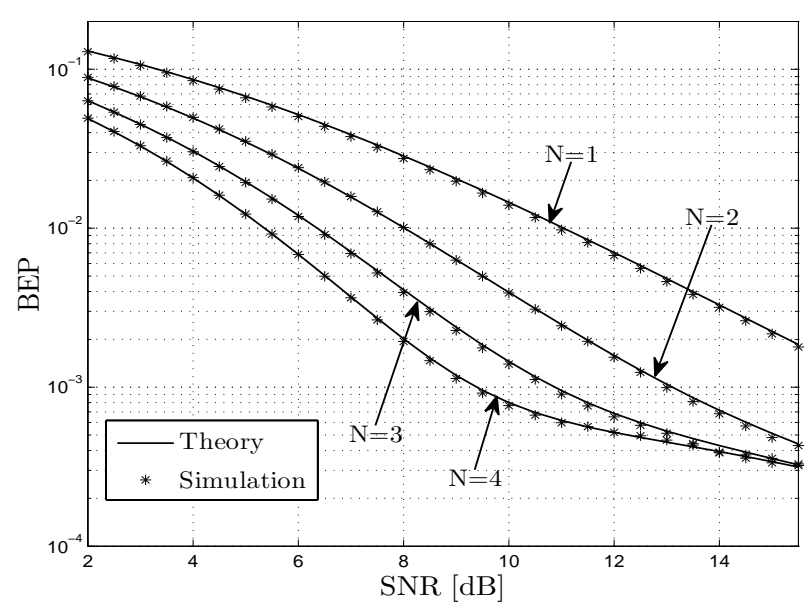

Fig. 3. BEP versus SNR of the SMIR scheme for different numbers of relays $(\xi=5 \mathrm{~dB}$, BPSK modulation).

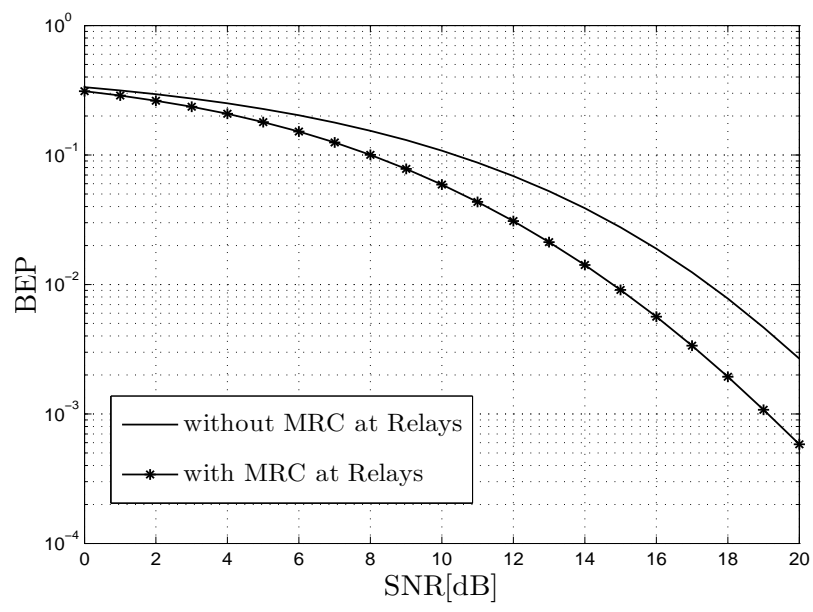

Fig. 4. BEP versus SNR of the SMIR scheme with MRC at relays and without MRC at relays ( $\xi=5 \mathrm{~dB}$, BPSK modulation).

\section{Average Number of Time Slots Per Burst}

\section{A. General Expression}

In this section, we present the exact expression for the average number of time slots $\overline{N_{p}}$ required to send a data burst, where $N_{p}$ is a random variable. Given a network state, the number of time slots $N_{p}$ required to send an arbitrary data burst has the following probability distribution

$$
\begin{aligned}
& \operatorname{Pr}\left[N_{p}=1\right]=\operatorname{Pr}\left(\gamma_{S D}>\xi\right) \\
& \operatorname{Pr}\left[N_{p}=l \mid S_{l-1}=B_{i, l-1}\right]=\operatorname{Pr}\left(\gamma_{l-1}>\xi, \gamma_{l-2}<\xi\right), \\
& \quad \quad \text { if } \quad 2 \leq l \leq N \\
& \operatorname{Pr}\left[N_{p}=N+1 \mid S_{N}=B_{i, N}\right]=\operatorname{Pr}\left(\gamma_{N-1}<\xi\right) .
\end{aligned}
$$

The average number of time slots per burst can be computed with the law of total probability as follows

$$
\begin{aligned}
\overline{N_{p}} & =\sum_{l=1}^{N+1} l \sum_{i=0}^{2^{l-1}-1} \operatorname{Pr}\left[N_{p}=l \mid S_{l-1}=B_{i, l-1}\right] \times \\
& E\left(\operatorname{Pr}\left(S_{l-1}=B_{i, l-1} \mid \text { phase }=l\right)\right) \\
& =\sum_{l=1}^{N+1} \sum_{i=0}^{2^{l-1}-1} l \operatorname{Pr}\left[N_{p}=l \mid S_{l-1}=B_{i, l-1}\right] \prod_{k=1}^{l-1} E\left(P_{k, i}^{l}\right),
\end{aligned}
$$

where $E\left(P_{k, i}^{l}\right)$ is given by (18).

\section{B. Numerical Examples}

In this section, the analytical results presented in subsection IV.A are evaluated numerically and validated by Monte Carlo simulations. As a numerical example, Fig. 5 depicts the average number of time slots $\overline{N_{p}}$ to send a data burst versus SNR for different threshold values $\xi$. It can be seen from this figure that as the SNR increases the average number $\overline{N_{p}}$ of time slots per burst decreases. Thus, for the proposed SMIR scheme, only some relays in the network should be active while the others remain idle. This is a significant advantage compared to schemes without incremental relaying, such as the SMR scheme. Actually, when the SNR is high, it is wasteful to make all relays forward the message. It can be concluded from Fig. 5 that for a fixed SNR value, the average number of time slots per burst increases as $\xi$ increases. Hence, the choice of a high value of $\xi$ must be justified to save channel resources.

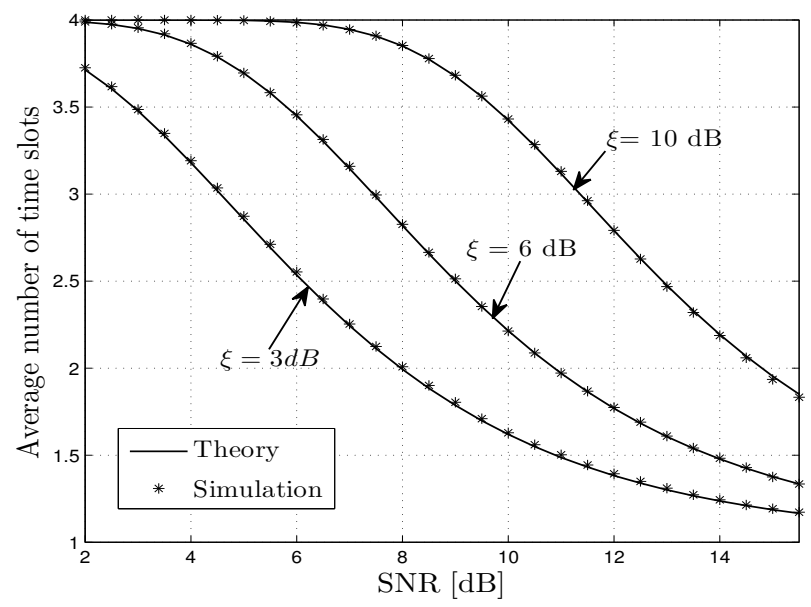

Fig. 5. Average number of time slots per burst versus SNR for different threshold values. The number of relays equals to 3 and the modulation scheme is BPSK.

Finally, Fig. 6 illustrates the BEP and the average number of time slots per burst versus $\xi$ for different constant values of the SNR. In our scenario, we consider a number of relays equal to 3 . In the first sub-figure, it can be observed that for a given SNR value there exists $\xi_{0}$ such that if $\xi \geq \xi_{0}$ the BEP reaches an error floor. For example, for an $\mathrm{SNR}=10 \mathrm{~dB}$, the BEP cannot drop below $10^{-3}$ if $\xi \geq \xi_{0}=7 \mathrm{~dB}$. This point $(\xi=7 \mathrm{~dB})$ corresponds to an average number of time slots per 
burst equals to 2.5 (out of 4 ). Thus, if we set $\xi>\xi_{0}$, we would have to use more transmission time slots compared to the case $\xi=\xi_{0}$ but without improving the BEP performance. This fact shows the advantage of the SMIR scheme. With this scheme, we do not need to use all network nodes (average number of time slots $=4$ ), but we utilize only an average number of relays equal to 2.5 without any loss in BEP performance. In addition, the threshold value in which BEP stabilizes depends on SNR value in which the system operates. For instance, if the $\mathrm{SNR}=8 \mathrm{~dB}$, the BEP cannot drop below $410^{-3}$. This BEP performance is achieved if $\xi \geq \xi_{0}=5 \mathrm{~dB}$. Note as well that the value of $\xi_{0}$ increases as the SNR increases. For example, if the SNR is set to $8 \mathrm{~dB}, 10 \mathrm{~dB}$, and $12 \mathrm{~dB}$ then $\xi_{0}$ equals to $5 \mathrm{~dB}, 7 \mathrm{~dB}$, and $8 \mathrm{~dB}$, respectively. By setting the value of $\xi$ equal to $\xi_{0}$ we guarantee that the best possible BEP performance as well as the optimal spectral efficiency are achieved.

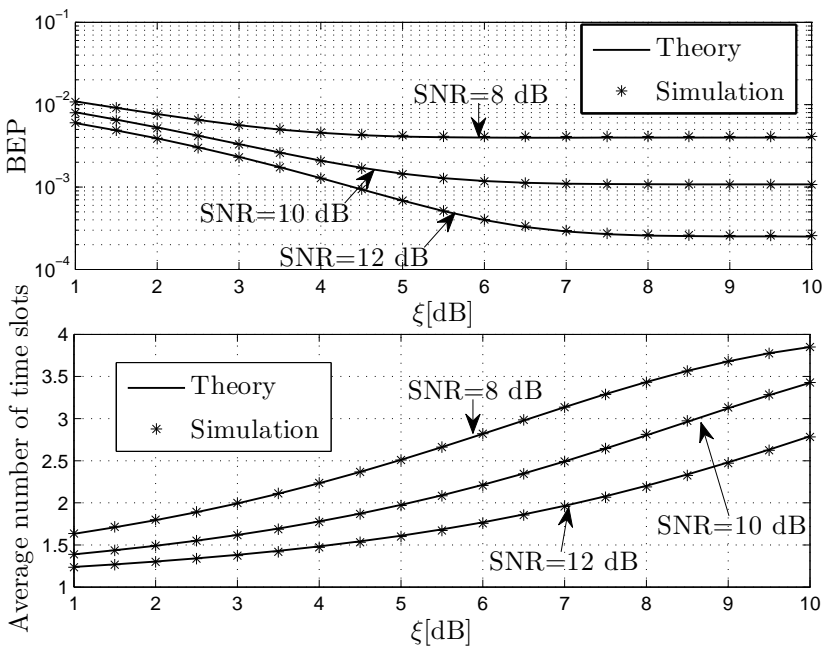

Fig. 6. BEP and average number of time slots per burst versus $\xi$ for constant values of SNR. The number of relays equals to 3 and the modulation scheme is BPSK.

\section{CONCLUSION}

In this paper, we proposed the relaying SMIR scheme and studied its performance. We derived exact expressions for the SEP over Rayleigh fading channels and evaluated the average number of time slots $\overline{N_{p}}$ required to send a data burst. The BEP of the proposed scheme is upper-bounded by the BEP of the direct link and lower-bounded by the BEP of the SMR scheme. We noticed that at low SNR values the proposed SMIR scheme and the SMR scheme have the same BEP. We demonstrated as well that the use of MRC at the relays allows achieving approximately a $3 \mathrm{~dB}$ gain compared to the case where no MRC is utilized at the relays.

Based on our analysis for the average number of time slots utilized to transmit a message successfully, we showed that the proposed protocol allows improving the spectral efficiency thanks to incremental relaying. Actually, at high SNR, the number of time slots used to transmit a message can be reduced significantly compared to schemes without incremental relaying, such as the SMR scheme. Moreover, we found that for a fixed SNR value, the proposed SMIR scheme can achieve the same BEP performance as the SMR scheme but with less average number of time slots. For the proposed SMIR scheme, a good BEP performance is obtained in the low SNR region and a gain in number of time slots is obtained especially at the high SNR regime.

\section{APPENDIX}

We present in this appendix the proof of (24). First, let us compute $f_{\gamma_{l}}\left(\gamma, \gamma_{l-1}<\xi\right)$. We discuss two intervals: $\gamma<\xi$ and $\gamma>\xi$.

- If $\gamma<\xi$,

$f_{\gamma_{l}}\left(\gamma, \gamma_{l-1}<\xi\right)=f_{\gamma_{l}}(\gamma)=f_{\gamma_{l}}(\gamma)=\sum_{j=0}^{m} \frac{C_{j}}{\bar{\lambda}_{j}} \exp \left(-\frac{\gamma}{\bar{\lambda}_{j}}\right)$

That is, $\gamma_{l}<\xi$ implies $\gamma_{l-1}<\xi$

- If $\gamma>\xi$, we notice that $\gamma_{l}=\gamma_{l-1}+\gamma_{R_{l} D}$. Since $\gamma_{l-1}$ and $\gamma_{R_{l} D}$ are independent random variables, so the PDF of $\gamma_{l}$ can be written as

$$
f_{\gamma_{l}}(\gamma)=\int_{0}^{\infty} f_{\gamma_{R_{l} D}}(\gamma-x) f_{\gamma_{l-1}}(x) d x
$$

Thus, the PDF of $f_{\gamma_{l}}\left(\gamma, \gamma_{l-1}<\xi\right)$ can be computed as

$$
\begin{gathered}
f_{\gamma_{l}}\left(\gamma, \gamma_{l-1}<\xi\right)=\int_{0}^{\xi} f_{\gamma_{R_{l} D}}(\gamma-x) f_{\gamma_{l-1}}(x) d x \\
=\sum_{j=0}^{m-1} \frac{C_{j}}{\bar{\lambda}_{j}}\left(\exp \left(-\xi\left(\frac{1}{\overline{\lambda_{j}}}-\frac{1}{\overline{\lambda_{m}}}\right)\right)-1\right) \times \\
\exp \left(-\frac{\gamma}{\overline{\lambda_{m}}}\right) \mathbf{1}_{\left(B_{i, l}[l]=1\right)}
\end{gathered}
$$

where $f_{\gamma_{l}}(\gamma), f_{\gamma_{l-1}}(\gamma)$ are given by (19) and $f_{\gamma_{R_{l} D}}(\gamma)$ is an exponential distribution.

\section{REFERENCES}

[1] J. Laneman, D. Tse, and G. Wornell, "Cooperative diversity in wireless networks: efficient protocols and outage behavior," IEEE Transactions on Information Theory, vol. 50, no. 12, pp. 3062-3080, Dec. 2004.

[2] A. Sendonaris, E. Erkip, and B. Aazhang, "User cooperation diversitypart I: system description," IEEE Transactions on Communications, vol. 51, no. 11, pp. 1927-1938, Nov. 2003.

[3] — , "User cooperation diversity-part II: implementation aspects and performance analysis," IEEE Transactions on Communications, vol. 51, no. 11, pp. 1939-1948, Nov. 2003.

[4] J. Boyer, D. Falconer, and H. Yanikomeroglu, "Multihop diversity in wireless relaying channels," IEEE Transactions on Communications, vol. 52, no. 10, pp. 1820-1830, Oct. 2004.

[5] M. Hasna and M.-S. Alouini, "Outage probability of multihop transmission over nakagami fading channels," IEEE Communications Letters, vol. 7, no. 5, pp. 216-218, May 2003.

[6] P. Anghel and M. Kaveh, "Exact symbol error probability of a cooperative network in a rayleigh-fading environment," IEEE Transactions on Wireless Communications, vol. 3, no. 5, pp. 1416-1421, Sep. 2004.

[7] J. Laneman and G. Wornell, "Distributed space-time-coded protocols for exploiting cooperative diversity in wireless networks," IEEE Transactions on Information Theory, vol. 49, no. 10, pp. 2415-2425, Oct. 2003.

[8] G. Amarasuriya, M. Ardakani, and C. Tellambura, "Output-threshold multiple-relay-selection scheme for cooperative wireless networks," IEEE Transactions on Vehicular Technology, vol. 59, no. 6, pp. 30913097, Jul. 2010 
[9] W. Choi, D. I. Kim, and B.-H. Kim, "Adaptive multi-node incremental relaying for hybrid-ARQ in AF relay networks," IEEE Transactions on Wireless Communications, vol. 9, no. 2, pp. 505-511, Feb. 2010.

[10] A. Sadek and K. Liu, "Multinode cooperative communications in wireless networks," IEEE Transactions on Signal Processing, vol. 55, no. 1, pp. 341-355, Jan. 2007.

[11] T. Nechiporenko, P. Kalansuriya, and C. Tellambura, "Performance of optimum switching adaptive $\mathrm{m}$-qam for amplify-and-forward relays," IEEE Transactions on Vehicular Technology, vol. 58, no. 5, pp. 22582268, 2009.

[12] M. Simon and M. Alouini, "A unified approach to the performance analysis of digital communication over generalized fading channels," Proceedings of the IEEE, vol. 86, no. 9, pp. 1860-1877, Sep. 1998.

[13] J. G. Proakis, Digital Communications, 4th ed. McGraw-Hill, 2001.

[14] S. S. Ikki and M. H. Ahmed, "Performance analysis of decodeand-forward incremental relaying cooperative-diversity networks over rayleigh fading channels," in Proc. 69th IEEE Vehicular Technology Conference (VTC-Spring 2009), Barcelona, Spain, Apr. 2009, pp. 1-6. 